\title{
The influence of cyano compound on liquid crystal blue phase range
}

\author{
O. Chojnowska, ${ }^{*}$ R. Dąbrowski \\ ${ }^{1}$ Institute of Chemistry, Military University of Technology, Kaliskiego 2, 00-908 Warsaw
}

Received May 29, 2012; accepted June 28, 2012; published June 30, 2012

\begin{abstract}
A liquid crystal blue phase (BP) is interesting for fast light modulators and tunable photonic crystals. In this study we investigated the influence of mixture composition on thermal stability of BP, especially the influence of cyano compound addition. The relationship between polymer stabilization possibility and cyano dopant was also examined. It was shown that the cyano compound is not necessary to create a blue phase and it has no effect on polymer stabilization of a blue phase.
\end{abstract}

The study on a liquid crystal blue phase has been rapidly extended since Kikuchi and coworkers [1] published the results of research on a polymer-stabilized blue phase. A blue phase usually exists in a very narrow temperature range (from $0.1 \mathrm{~K}$ to $5 \mathrm{~K}$ ) in one or a few types: BPI, BPII and BPIII. There are several methods to extend thermal stability: adding nanoparticles or bentshaped molecules and creating a polymer network. It is considered that amorphous polymer chains or nanoparticles are selectively concentrated in disclination lines to stabilize the lattice structure of blue phases $[1,2]$. A different mechanism is observed in systems containing bent-shaped molecules. Molecular simulations show us that these molecules enhance a blue phase temperature range by taking free places in the space between two helices [3].

It is proposed that a blue phase has a periodic cubic lattice of double twist tubes. The BP consists of a cylindrical double twist tube in which the director rotates spatially about any radius of a cylinder and is parallel to the cylinder axis at the centre of the cylinder [4]. Matching these tubes in three dimensional space is impossible and affects disclinations - defects in the arrangement of molecules. A blue phase occurs whenever there is a balance between defects and a local energetic minimum of the director, being a frustrated system.

According to Sethna [5], in frustrated systems different terms in free energy dictate different molecular arrangements, and the competition between them results in a more complex structure. Defects in a blue phase make the structure less stable so it is energetically favorable only in a narrow temperature range. The structure of BPI and BPII was identified by many experiments and theory calculation [6]. BPI has bodycentred cubic symmetry, BPII has a simple cubic structure

*E-mail: o.chojnowska@wat.edu.pl and BPIII is amorphous with a short distance order of double twist alone.

A blue phase exhibits several revolutionary features in comparison to conventional nematic materials. Firstly, it does not require any alignment layer, such as polyimide, which simplifies the manufacturing process and reduces the cost. Secondly, the dark state of a blue-phase liquid crystal display (LCD) is optically isotropic so that its viewing angle is wide and symmetric. Anisotropy appears when an external electric field is applied, which results in splitting the light beam passing through the sample at an angle varying from the direction of an electric charge flow. This phenomenon is called the electrooptic Kerr effect and is affected by operating voltage. The higher is the Kerr constant $(K)$, the smaller is on-state voltage $\left(V_{o n}\right)$ required. In order to extend this parameter the host liquid crystal composite should preferably have a high birefringence $(\Delta n)$, large dielectric anisotropy $(\Delta \varepsilon)$, long pitch length $(P)$, and small elastic constant $(k)$ [7], which is expressed by the following equation:

$$
K \sim \frac{\delta n}{\lambda E^{2}} \approx \Delta n \cdot \Delta \varepsilon \frac{\varepsilon_{0} P^{2}}{k \lambda(2 \pi)^{2}}
$$

The electro-optic response times rise, as well as decay times are lower than one millisecond [8]. The magnitude of response time is proportional to rotational viscosity $\left(\gamma_{1}\right)$, square of the helical pitch $(P)$, inversely proportional to the elastic constant $(k)$ [9], which can be presented by the following equation:

$$
\tau \approx \frac{\gamma_{1} P^{2}}{k(2 \pi)^{2}}
$$

The response time is independent of the cell gap in contrast to other liquid crystal devices and this feature is attractive, especially for devices designed for the $\mathrm{THz}$ and $\mathrm{GHz}$ frequency range of electromagnetic radiation.

There are, however, a few remaining challenges that need to be met: hysteresis, residual birefringence and too high operating voltage. A fast response time at relatively low voltages cannot be obtained by the helix pitch variation, because the response time is proportional to the helical pitch and on-state voltage is inversely proportional to its value [see Eq. (1)-(2)]. 
Commonly known systems exhibiting BP are based on commercial mixtures diluted with 4-cyano-4'pentylbiphenyl (5CB) and doped with chiral compounds $[10,11]$. According to the literature it is not clear what chemical structures are preferred for the occurrence of blue phases. This letter shows the results of investigation of cyano compounds (5CB) influence on blue phase occurrence and temperature range. It was examined if the presence of cyano compounds is necessary for blue phase stabilization or whether it may be observed without them. This fact has practical aspects because in the systems addressed by active matrices mainly fluorinated compounds are used. They exhibit the necessary high resistivity, high photochemical stability and also low viscosity. The relationship between possible polymer stabilization and cyano dopant occurrence was also examined here.

The liquid crystal material used in this study was the nematic mixture 1855 prepared by us and consisting of three compounds of a homologous series of 3,4-difluoro4'-(4-alkylcyclohexyl)biphenyls (formula 1) (from Valiant Fine Chemicals) shown in Table 1. Dielectric and optical anisotropy of base mixture 1855 are $\Delta \varepsilon=7.06$ (measured at $T=298 \mathrm{~K}$ and $f=1 \mathrm{kHz}$ ) and $\Delta n=0.1481$ (measured at $T=298 \mathrm{~K}$ and $\lambda=589 \mathrm{~nm}$ ). The temperatures of phase transitions are $\mathrm{Cr} 15 \mathrm{~N} 94$ Iso.

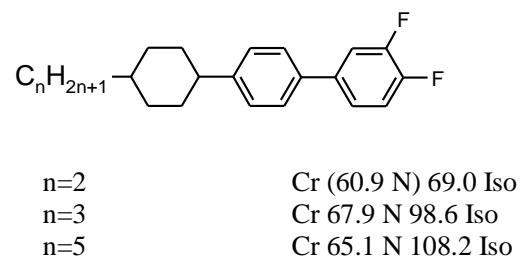

Table 1. General formula and temperatures of phase transitions of nematic base 1855 components.

To induce a blue phase a chiral dopant [1,1';4',1']terphenyl-4,4"'-dicarboxylic acid bis-(1methylheptyl) ester (formula 3 ) was used. This compound was synthesized in our laboratory by Kula [12]. The helical twisting power (HTP) of this optically active compound measured in a cholesteric phase of base mixture 1855 was $25 \mu^{-1}$. Its HTP was also measured in a mixture with negative dielectric anisotropy and can be found in [13]. The monomers used in this study to form a polymer network in the composite systems were dodecyl acrylate (formula 4) and 1,4-bis-[4-(3acryloyloxypropoxy) benzoyloxy]-2-methylbenzene (formula 5) (from Chemos $\mathrm{GmbH}$ ). The photoinitiator 2,2-dimethoxy-2-phenylacetophenone (formula 6) (from Sigma-Aldrich) was added. This initiator has three main absorption bands, one of them with a maximum at $341 \mathrm{~nm}$ [14] has part of the band in a wavelength range which is transmitted through the glass $(350-365 \mathrm{~nm})$. Chemical structures of all compounds of investigated systems are given in Table 2. Prepared mixtures were put into a glass cell constructed from two glass substrates separated by spacers with a diameter of $10 \mu \mathrm{m}$. The optical textures of the blue phases and their range were observed by a polarizing optical microscope OLYMPUS BX51 equipped with a LINKAM THMS600 heating stage. The temperature was controlled with an accuracy of $\pm 0.1 \mathrm{~K}$.

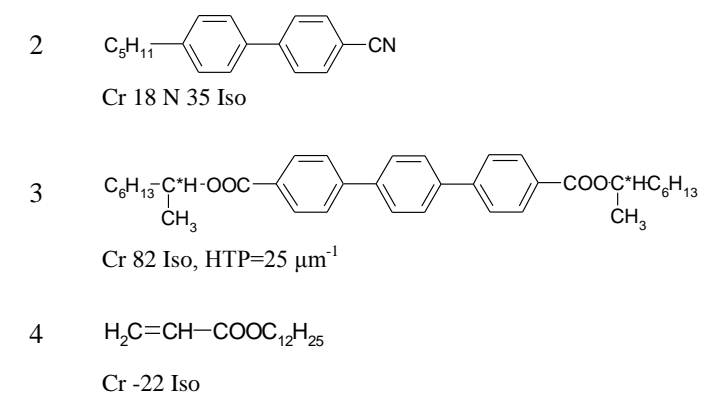

5

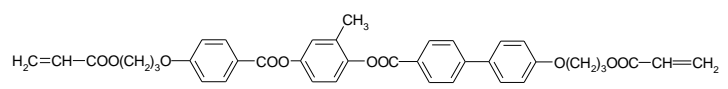
Cr 70 N 126 Iso

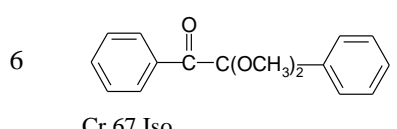

Table 2. Chemical structures of investigated system components and their temperature of phase transitions.

To compare blue phase thermal stability in a pure mixture and in a mixture diluted $5 \mathrm{CB}$, the nematic base 1855 was doped with a 0.2 mole fraction of $5 \mathrm{CB}$. The mole ratio of a chiral dopant in both systems was 0.1 . The results are shown in Table 3 . The temperature range of a blue phase obtained was narrower in a system with $5 \mathrm{CB}$ $\left(0.5^{\circ} \mathrm{C}\right.$ in heating and $3.3^{\circ} \mathrm{C}$ in cooling). In a mixture without $5 \mathrm{CB}$ the blue phase temperature range was almost two times wider $\left(0.9^{\circ} \mathrm{C}\right.$ in a heating and $6.9^{\circ} \mathrm{C}$ in a cooling cycle).

\begin{tabular}{|c|c|c|c|c|c|c|}
\hline 1 & 2 & $\begin{array}{l}3 \\
\mathrm{~mol}\end{array}$ & $\begin{array}{l}4 \\
\text { fractio }\end{array}$ & 5 & 6 & $\mathrm{~T}$ of phase transitions $\left[{ }^{\circ} \mathrm{C}\right]$ \\
\hline 0.9 & - & 0.1 & - & - & - & $\begin{array}{l}\mathrm{N}^{*} 71.3 \text { BPI } 71.7 \text { BPII } 72.2 \text { Iso }^{\text {a) }} \\
\text { Iso } 72.0 \text { BPII } 71.5 \text { BPI } 65.1 \mathrm{~N}^{*} \text { b) }\end{array}$ \\
\hline 0.7 & 0.2 & 0.1 & - & - & - & $\begin{array}{l}\mathrm{N}^{*} \text { 67.4 BPI } 67.8 \text { BPII } 67.9 \text { Iso a) } \\
\text { Iso } 67.8 \text { BPII } 67.5 \text { BPI } 64.5 \mathrm{~N}^{*} \text { b) }\end{array}$ \\
\hline 0.8 & - & 0.1 & 0.03 & 0.06 & 0.01 & $\begin{array}{l}\mathrm{N}^{*} 66.4 \text { BPI } 67.0 \text { BPII } 67.4 \text { Iso } \\
\text { a),c) } \\
\mathrm{N}^{*}<-30 \text { BPI ? BPII } 73.4 \text { Iso }^{\text {d) }}\end{array}$ \\
\hline 0.6 & 0.2 & 0.1 & 0.03 & 0.06 & 0.01 & $\begin{array}{l}\mathrm{N}^{*} \text { (c) } \\
\text { a),3 BPI } 51.0 \text { BPII } 51.1 \text { Iso } \\
\mathrm{N}^{*}<-30 \text { BPI ? BPII } 55.2 \text { Iso }{ }^{\text {d) }}\end{array}$ \\
\hline
\end{tabular}

Table 3. Composition of investigated systems and their temperatures of phase transitions in heating and cooling cycles. 

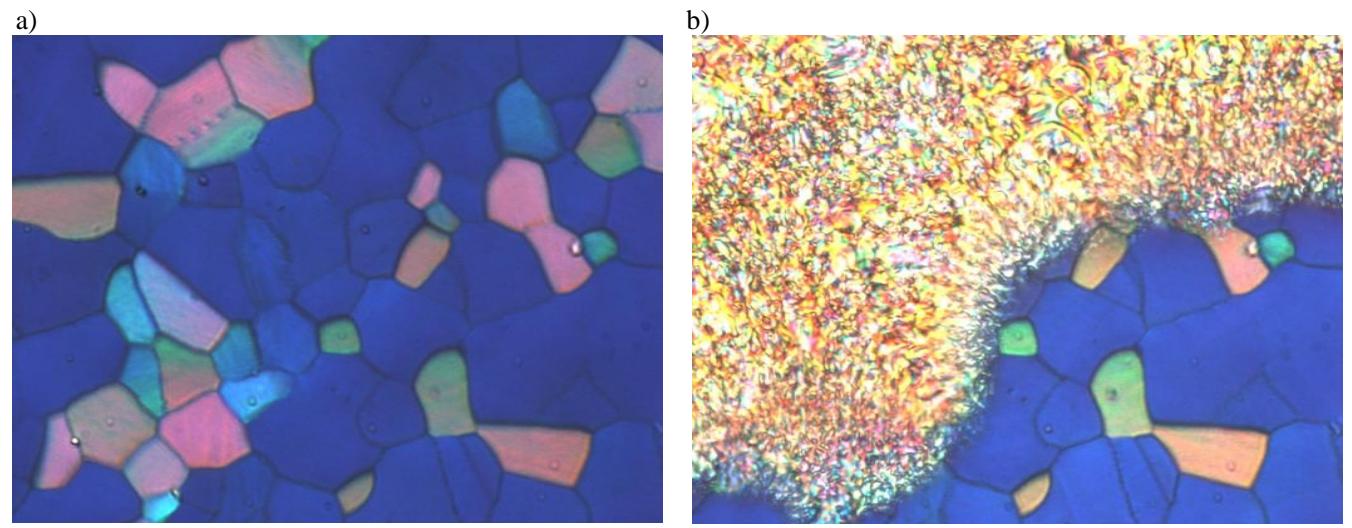

Fig. 1. Microscoping textures of a) BPI at $68^{\circ} \mathrm{C}$ and b) BPI-N* transition at $65.1^{\circ} \mathrm{C}$ in base mixture 1855 doped with 0.1 mole fraction of the chiral dopant, observed in cooling cycle.

As we can see, the addition of 4-cyano-4'pentylbiphenyl to a mixture containing fluorinated compounds reduced the stability of BP. The range of BP became narrower and temperature of $\mathrm{N}^{*}$-BPI transition decreased. This effect results from a lower clearing point of compound 2.The textures of a blue phase obtained in a cooling cycle in mixture 1855 doped with a chiral dopant are given in Fig. 1.

The relationship between possible polymer stabilization and cyano compound presence was examined, too. To prepare composites by forming an amorphous polymer network in the liquid crystal blue phase, monofunctional monomer (4), difunctional monomer (5) and photoinitiator (6) were added to the mixtures with a blue phase. These mixtures were the same as in the first part of the experiment: the base mixture 1855 doped with a 0.1 mole fraction of chiral dopant (3) and mixture 1855 doped with a 0.2 mole fraction of $5 \mathrm{CB}$ and a 0.1 mole fraction of (3). The fractions of the monomers are shown in Table 3 . The homogenous mixture of liquid crystals, monomers and photoinitiator were prepared by stirring in an isotropic phase. Then it was put into a glass cell $10 \mu \mathrm{m}$ thick, followed by irradiation for 30 minutes with UV light in a supercooled state (at $0.8 \mathrm{~K}$ below the temperature of $\mathrm{N}^{*}$-BPI transition measured in heating). The density power was 0.2 $\mathrm{mW} / \mathrm{cm}^{2}$ measured at $365 \mathrm{~nm}$ (the light source was metal halide lamp Hamamatsu Photonics LC8). After polymerization the cell was cooled with the rate of temperature change $1 \mathrm{~K} / \mathrm{min}$.

The polymer stabilizing effect was observed in both systems. In a mixture containing $5 \mathrm{CB}$ the $\mathrm{BP}$ range was observed by more than $70 \mathrm{~K}$ but the temperature of BPIIIso transition was lower than in a mixture without a polymer network. An opposite phenomenon was observed in a mixture without $5 \mathrm{CB}$. The clearing point was higher in a polymer-stabilized system and the BP range was wider than $100 \mathrm{~K}$. The presence of $5 \mathrm{CB}$ is not necessary for polymer stabilization because it decreases the clearing point and complicates the stabilization process. In the early stages of polymerization, the temperatures of phase transition are likely to shift so it is required to use a material with as wide BP temperature range as possible.

In conclusion, a blue phase can be formulated and enhanced by a polymer network in mixtures composed of only fluorinated compounds, which is important from the application point of view. Fluorinated compounds have higher resistivity and photochemical stability than cyano compounds.

This work was supported by the National Centre for Research and Development under the grant PBS826. The authors thank professor Shin-Tson $\mathrm{Wu}$ from the University of Central Florida, for providing the monofunctional monomer: dodecyl acrylate.

\section{References}

[1] H. Kikuchi, M. Yokota, Y. Hisakado, H. Yang, T. Kajiyama, Nature Materials 1, 64 (2002).

[2] H. Yoshida, Y. Tanaka, K. Kawamoto, H. Kubo, T. Tsuda, A. Fuji, S. Kuwabata, H. Kikuchi, M. Ozaki, Appl. Phys. Expr. 2, 12501 (2009).

[3] K.Z. Zheng, D. Shen, P. Huang, New J. Phys. 12, 113018 (2010).

[4] H. Kikuchi, H. Higuchi, SID07 Digest, 38, 1737 (2007).

[5] J.P. Sethna, in Theory and Applications of Liquid Crystals (edited by J. L. Ericksen and D. Kinderlehrer; Springer-Verlag 1987).

[6] P.P. Crooker, in Chirality in Liquid Crystals (edited by H.S. Kitzerow and C. Bahr; Springer, New York 2001).

[7] P.R. Gerber, Mol. Cryst. Liq. Cryst. 116, 197 (1985).

[8] K.M. Chen, S. Gauza, H. Xianyu, S.T. Wu, J. Display Technol. 6, 49 (2010).

[9] S.T. Wu, D.K. Yang, Reflective Liquid Crystal Displays (New York, Wiley 2001).

[10] S.K. Hong, G.L. Lim, H. Kikuchi, Mol. Cryst. Liq. Cryst. 511, 248 (2009).

[11] S.K. Hong, H.S. Choi, S. Shibayama, H. Higuchi, H. Kikuchi, Mol. Cryst. Liq. Cryst. 528, 32 (2010).

[12] P. Kula, Synthesis and properties of terphenyl and quaterphenyl based chiral diesters; Liquid Crystals, in press

[13] W. Rejmer, K. Czupryński, W. Drzewiński, Mol. Cryst. Liq. Cryst. 547, 33 (2011).

[14] H. Kaczmarek, P. Gałka, Open Proc. Chem. J. 1, 8 (2008). 